Published by Al-Nahrain College of Medicine P-ISSN 1681-6579

E-ISSN 2224-4719

Email: iraqijms@colmed-alnahrain.edu.iq http://www.colmed-alnahrain.edu.iq http://www.iraqijms.net

\title{
The Efficiency of Molecular and Conventional Methods in Detection of Candida albicans Isolated from Immunocommpromsed Patients with Pulmonary Symptoms
}

\author{
Azhar A.F. Al-Attraqchi ${ }^{1} P h D$, Marwa A. Hadab $^{1} M S C, F A B M$, Jabbar S. Hassan ${ }^{1} P h D$, Haider N. \\ Dawood $^{2}$ FIBM
}

${ }^{1}$ Dept. of Medical Microbiology, College of Medicine, Al-Nahrain University, Baghdad, Iraq, ${ }^{2}$ Dept. of Medicine, Imamein Kadhimein Medical City, Baghdad, Iraq.

\begin{abstract}
Background Candida albicans (C. albicans) has emerged as a potentially pathogenic fungus rather than benefit mucosal commensal in patients with pulmonary diseases. Although respiratory candidiasis secondary to pulmonary tuberculosis has been reported in the past, it has gained more relevance recently due to increased use of broad spectrum antibiotics and immunosuppressive drugs.

Objective To detect $C$. albicans in sputum samples from patients with pulmonary diseases using conventional and molecular methods.

Methods One hundred sputum samples obtained from patients with pulmonary symptoms were included in this study. Sputum samples were dispensed into three specimen parts; the first one was applicated for cultured on Sabouraud dextrose agar at $37^{\circ} \mathrm{C}$ for $48 \mathrm{hrs}$ and then the purified colony of Candida underwent biochemical tests including API Candida strips, and germ tube. The second part was undergone direct gram stain, while the third part was applicated for DNA extraction and then molecular diagnosis with PCR technique using specific primers.

Results Culture result revealed 43 positive samples for Candida species out of 100 samples. Among these positive samples, $23(53.5 \%)$ were positive for $C$. albicans in each of culture, germ tube and API. Molecular test revealed an amplicon with 538bp fragment of phospholipase gene from the same 23 samples.

Conclusion C. albicans is highly prevalent among patients suffering from bronchopulmonary symptoms. The molecular and conventional methods gave concomitant results as detection tools for the diagnosis of such microorganisms.

Keywords Candida albicans, phospholipase B gene, sputum.
\end{abstract}

DOI: 10.22578/IJMS.14.4.6

List of abbreviation: $C=$ Candida, PLB $=$ Phospholipase $B, S A S=$ Statistical analysis system, SDA = Sabouraud's Dextrose agar

\section{Introduction}

andidiasis is secondary mycotic infection caused by members of the genus Candida. Chiefly, Candida albicans (C. albicans) is responsible for about 70 to $80 \%$ of all Candida infections. Infection with $C$. albicans, which is an opportunistic yeast pathogen, increases predominantly in patients with predisposing condition including immunodeficiency such as human immune deficiency virus infections, prolong used of broad-spectrum antibiotics, corticosteroids therapy, diabetic patients and infections with other debilitated disease ${ }^{(1)}$.

In immunocompromised patients, the clinical appearance of the $C$. albicans infection is often very complex and identification of the 
organism is difficult. Therefore, speedy diagnosis and management of candidiasis are crucial for these patients ${ }^{(2)}$. C. albicans has the ability to form germ tube and chlamydospore, which is a characteristic feature of the yeast. $C$. albicans produces germ tubes when inoculated in serum and incubated for $30 \mathrm{~min}$ at $37^{\circ} \mathrm{C}$. The yeast cells have the ability to form germ tube in their initial stages when the hyphae are still attached to the yeast cell looking like sprouts ${ }^{(3)}$. Furthermore, this fungus secretes many enzymes such as proteinase, which has the ability to degrade a number of important defensive host proteins, particularly immunoglobulin and complement ${ }^{(4)}$.

Candida pneumonia is one of the most challenging infections of all the Candida infection. Pneumonia due to infection with Candida spp. is extremely rare, but because of contamination with oral flora, these organisms are frequently cultured from respiratory secretions ${ }^{(5,6)}$. Non-culture-based methods, such as DNA detection by PCR, have been developed in order to assist in the rapid diagnosis of fungal infections, allowing for the initiation of species-oriented therapy as early as $6 \mathrm{~h}$ after the onset of disease ${ }^{(7)}$.

This study was carried out to detect $C$. albicans in sputum samples from patients with pulmonary diseases using conventional and molecular methods.

\section{Methods}

Sputum samples have been collected from 100 patients of age group ranged from 10-90 years old, with a mean age $47.23 \pm 19.51$. Some of these patients were suffering from systematic disease such as such as tuberculosis, diabetes mellitus, leukemia, while other were with immunocompromised status. Those patients were attending and admitting to Al-Yarmouk Teaching Hospital, Imamein Kadhimein Medical City and Chest and Respiratory Diseases Institute, Baghdad City Hospital during the period from September 2015 to February 2016. Each sputum sample was dispensed into three specimen parts the first one was applied for culture on Sabouraud dextrose agar at $37{ }^{\circ} \mathrm{C}$ for $48 \mathrm{hrs}$. Purified colonies from this culture were underwent biochemical tests including API Candida strips and germ tube. The second part was used in direct gram stain, while the third one was applied for molecular method. Standard strains of C. albicans ATCC 10231, was obtained from the National Institute of Health in Baghdad, which was used as a positive control.

\section{Isolation and identification of $\boldsymbol{C}$. albicans}

Gram stain method was applied to each fresh sputum specimen and examined microscopically for detecting Candida species. Sputum samples were streaked on Sabouraud's Dextrose Agar (SDA) and incubated at $37{ }^{\circ} \mathrm{C}$ for 24-48 hrs. The isolates were re-identified by using API $20 \mathrm{C}$ AUX and germ tube production. API 20 C AUX was performed according to the manufacturer's instructions (Biomuriex, France) for the confirmatory identification of the $C$. albicans and other species. Germ tubes production is a diagnostic characteristic method for $C$. albicans. A small part of yeast colony to be tested was emulsified with $0.5 \mathrm{ml}$ of mammalian serum in a small test tube. The tube was incubated aerobically at $37{ }^{\circ} \mathrm{C}$ in an incubator for $2 \mathrm{hrs}$. A drop of the serum was removed to a slide and examined microscopically using the $x 10$ and $x 40$ objective lenses. A cylindrical filament originating from the blastoconidium without any constriction at the point of origin and without obvious swelling along the length of the filaments indicates a germ tube positive yeast ${ }^{(7)}$.

\section{Molecular method for diagnosis of $\boldsymbol{C}$. albicans}

DNA was extracted from each sample using PrepIT. MAX, DNA genoTek, purification kit (Canada) with modification by mixing $200 \mu$ of the sputum sediment with the $40 \mu$ of MAX lysis Reagent. The resultant suspension was undergone repeat freezing-thawing by subjecting the samples to liquid nitrogen for 5 minute followed by boiling for $3 \mathrm{~min}$ for five cycles ${ }^{(8)}$. The primers set used for the 
amplification of PLB genes of Candida was Forward 5'-TTGTGTTGCTACATCACCAAC-3' and reverse $5^{\prime}$-TTTGCTGGCAACTTGATTACC- $3^{\prime(9)}$ to produce a DNA fragment of $538 \mathrm{bp}$. The thermocycling conditions were as follows: after initial denaturation at $94{ }^{\circ} \mathrm{C}$ for $5 \mathrm{~min}, 30-$ cycle amplification profile consisted of $95^{\circ} \mathrm{C}$ for $30 \mathrm{~s}, 63^{\circ} \mathrm{C}$ for $35 \mathrm{~s}$ and $72^{\circ} \mathrm{C}$ for $1 \mathrm{~min}$ was adapted. Final elongation was carried out at 72 ${ }^{\circ} \mathrm{C}$ for $10 \mathrm{~min}$. PCR products were processed into a $1.5 \%$ (wt/vol) agarose gel (MerckGermany) at $120 \mathrm{mV}$ for $30 \mathrm{~min}$. A molecular marker (100 pb DNA ladder; Bioneer/Korea) was run concurrently. DNA bands were visualized and photographed under UV light after the gel was stained with ethidium bromide.

\section{Statistical analysis}

Statistical Analysis system (SAS) software was used for all statistical analysis continuous variables were expressed in mean \pm standard deviation (SD). The Pearson's Chi-square test or Fisher exact test was used for comparing the categorical variable. A two-sided significant level of 0.05 was considered to indicate a statistically significant difference.

\section{Results}

Demographic and clinical characteristics of patients:

The characteristics of the study population are shown in table (1).

\section{Albicans from patients with pulmonary} manifestation and other underlying diseases:

C. albicans was isolated from 4 (18.1\%), patients with hematological malignancies, 8 (28.5\%), patients with solid tumor, 1 (4.3\%), patients with asthma, $6(23.07 \%)$ patients with diabetes mellitus and $4(17.4 \%)$ patients with tuberculosis.

\section{Cultivation and Gram stain}

A total of 100 sputum samples were cultivated on Sabouraud's Dextrose agar and incubated for 2 days at $30{ }^{\circ} \mathrm{C}$. Forty three samples (43\%) were positive for Candida species the colonies were mucoid and have a creamy color (Figure 1). Gram stain confirmed this result in that the 43 samples were gram positive.

Table 1. Demographic and clinical features of patients

\begin{tabular}{cc}
\hline Variables & Value \\
\hline Age (mean \pm SD) & $(47.23 \pm 19.51)$ \\
Sex M:F (No.) & $64: 36$ \\
Clinical Features (No, \%) & \\
Hematological & $22(22 \%)$ \\
malignancies & $28(28 \%)$ \\
Solid tumors & $5(5 \%)$ \\
Asthma & $26(26 \%)$ \\
Diabetes mellitus & $33(33 \%)$ \\
Tuberculosis &
\end{tabular}

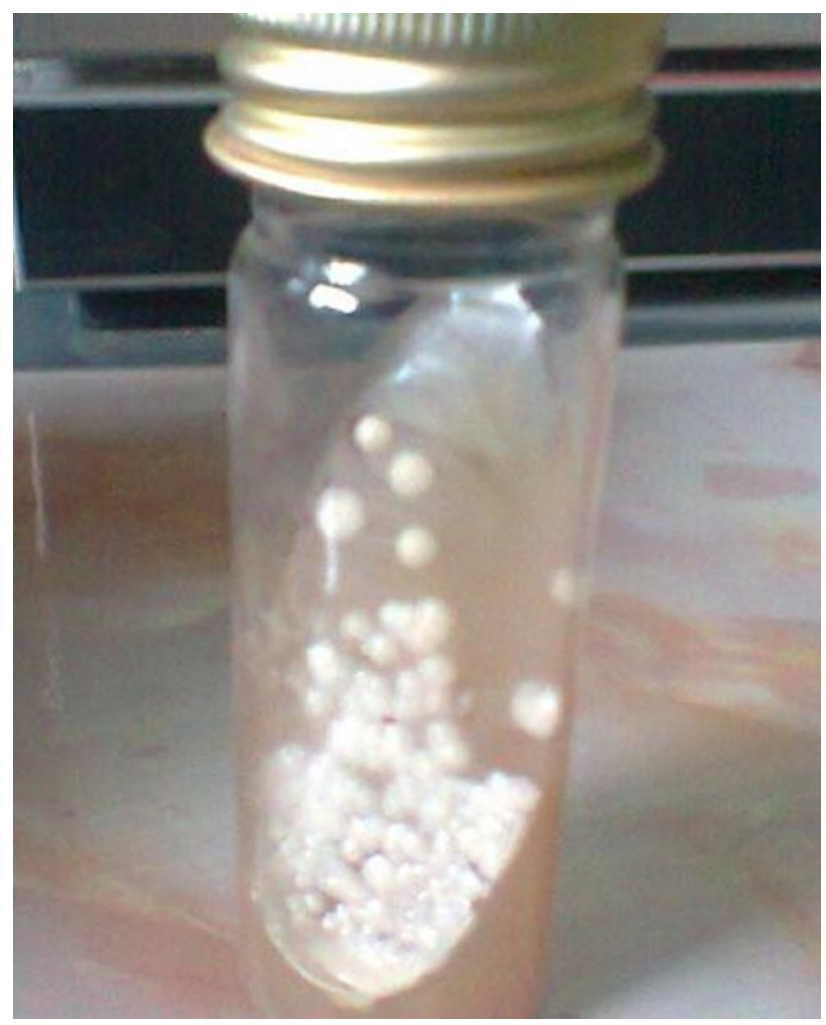

Figure 1. Candida species colonies on Sabouraud's agar media 


\section{Germ tube formation}

A total of 43 culture samples were examined for germ tube. The result revealed that 23
(53\%) were positive for C. albicans, as shown in Figure 2.

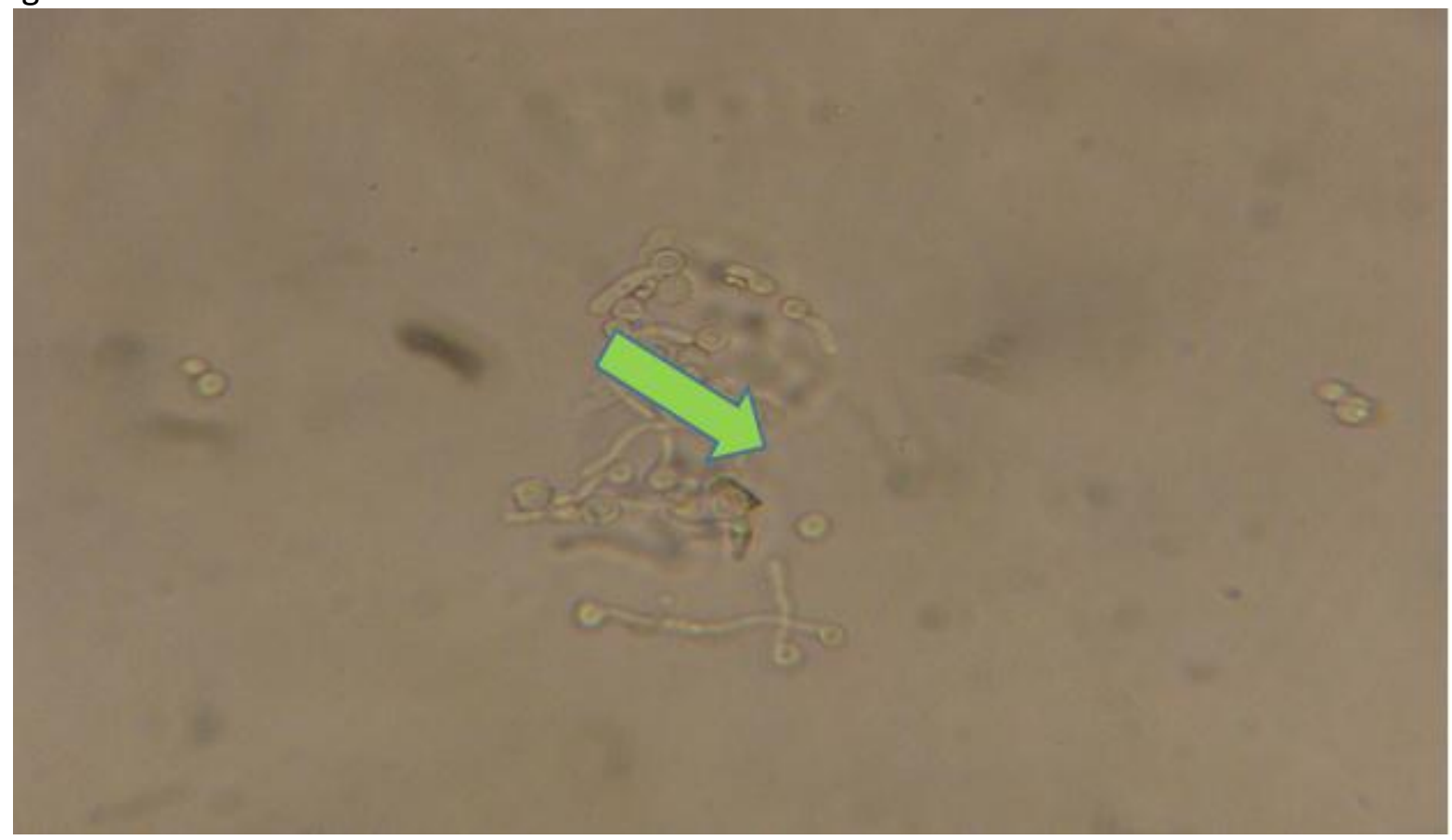

Figure 2. Germ tube formation by Candida albicans

\section{API Candida kit}

A total of 23 positive cultures were examined by API 20 AUX Candida strips. It was found that all samples represent C. albicans 23 (23\%). Using PCR as a golden standard to evaluate both germ tube and API Candida system, the specificity and sensitivity of both API Candida system and germ tube are $100 \%$.

\section{Molecular detection}

Conventional PCR was done for the amplification of PLB gene, which by using specific set of primers sequences. The results showed that, this gene (PLB gene) was present in twenty three out of one hundred sputum samples (23\%). PCR product of this gene was 538 bp. (Figure 3 ).

\section{Discussion}

In the present study, relatively high percentage of C. albicans infection was found among patients with hematological malignancies, solid tumor, asthma, diabetes mellitus and from patients with tuberculosis. These results are in accordance with those obtained by Ansari, et al (10) who showed that a fungal infection represents a growing problem in patients with hematologic malignancies. In particular, with chemotherapy-induced neutropenia, the majority of the infections were referred as $C$. albicans (74.7\%). 11. Ramirez-Garcia, et al (11) reported that, the opportunistic fungus $C$. albicans increases the risk of carcinogenesis and metastasis. In another's study, $C$. albicans was isolated from sever patients with diabetes mellitus ${ }^{(12)}$, while Kali et al ${ }^{(13)}$ mentioned that Candida co-infection was observed in $40 \%$ of patients with pulmonary tuberculosis.

Many factors are accused for this increment in fungal infections, the most important of which is massive and prolong use of immunosuppressive drugs. Most of these drugs are chemotherapies intended to treat malignancies. However, they reversely affect the immune system and eventually enhance the opportunistic microorganisms, to invade the body ${ }^{(14)}$. Beside chemotherapies, the 
invasive method for diagnosis or treatment in intensive care unit may facilitate the contamination with C. albicans. Other factors, like personal hygiene, also have a role ${ }^{(14)}$.

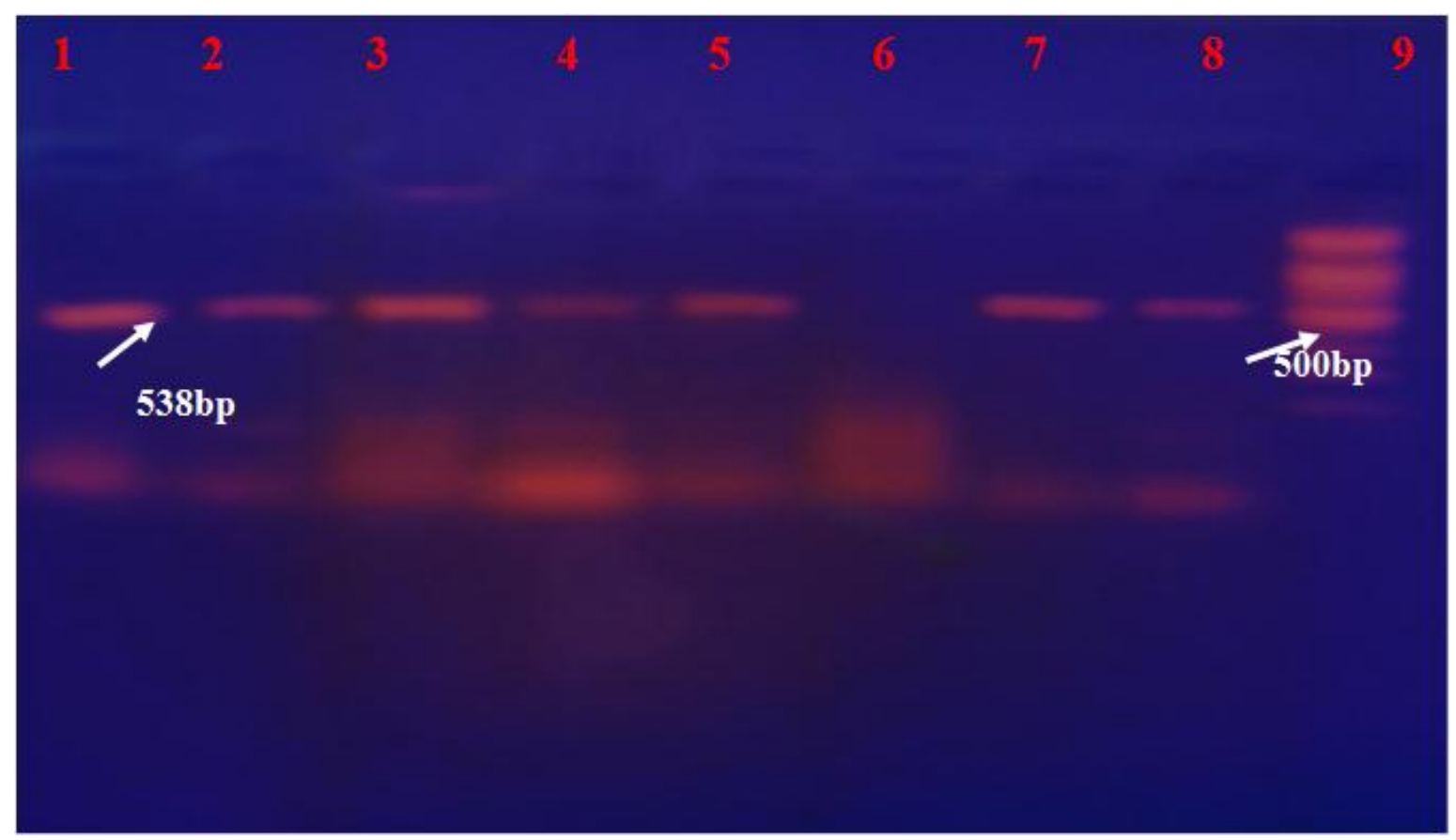

Figure 3. Gel electrophoresis (2\% agarose, 7v/cm2, 1.5hrs) of the PCR products, lane 9(MW): 100 bp DNA ladder; lane (1-5 \&7) Positive sample for (PLB genes of Candida albicans gene538 bp); lane 6: Negative control. Lane 8: Positive control

Microscopic examination of sputum using staining methods remains popular in the diagnosis of pulmonary infection especially in low-income countries, due to its rapidity, low cost, relatively easy to perform and high positive predictive value ${ }^{(15)}$. On the other hands, culture is considered to be the "gold standard" method for the diagnosis of pulmonary infections but require 20 to 100 viable organisms per sample and this is a cumbersome in partially treated patients. Culture also labor intensive and timeconsuming ${ }^{(16)}$.

In this study, positive cultures were tested by germ tube and biochemical API 20 AUX. Results of germ tube revealed that $53.4 \%$ of positive culture were $C$. albicans. All germ tube positive samples were positive for $C$. albicans by API 20 AUX. The API 20 AUX, and germ tube technique provides a convenient and reliable methods for identification of $C$. albicans.
Twenty three samples were positive for PLB gene, which is specific for $C$. albicans. The PLB gene of Candida species is a novel target, which shows a high variability of sequences among Candida. The nucleotide sequence variability between the different species of Candida can reach $95 \%{ }^{(17)}$. Thus, it is possible through designing specific set of primers to target the unique sequence of PLB gene.

Being used the same set of primers as in the current study Harmal et al (9) proved that species-specific PCR assay could identify and differentiate between the four most common Candida species isolated from clinical specimens namely, C. albicans, C. glabrata, $C$. parapsilosis and $C$. tropicalis. Distinctive product size for each of these 4 species allow specific identification directly from the gel electrophoresis without the need for further genotyping.

Based on molecular weight of amplicon product from that PCR product of this gene, it 
was 404 bp in Candida glabrata and 252 bp in C. parapslosis ${ }^{(18,19)}$.

In conclusion, Candida albicans is an important fungal infection formed a high percentage among patients suffering from bronchopulmonary symptoms. The molecular and conventional methods gave concomitant results as detection tools for the diagnosis of such microorganisms.

\section{Acknowledgments}

The author is grateful to all staff member of Medical Microbiology Department, College of Medicine, Al-Nahrain University for their help and cooperation.

\section{Author contribution}

Dr. Al-Attraqchi made the drafting of the article and revising it critically for important intellectual content. Dr. Dawood was responsible for samples and patients selections. Dr. Hassan made the DNA extractions, molecular, conventional methods diagnosis, analysis and interpretation of results and statistical analysis, while Hadab was responsible for the design and acquisition of data, molecular material supplementation.

\section{Conflict of interest}

The author declares that they have no competing interests.

\section{Funding}

Self-funding.

\section{References}

1. Estrada-Mata E, Navarro-Arias MJ, Pérez-García LA, et al. Members of the Candida parapsilosis Complex and Candida albicans are differentially recognized by human peripheral blood mononuclear cells. Frontiers Microbiol J. 2016; 6; 29-40.

2. Ryan KJ, Ray CG. Sherris Medical Microbiology - An introduction to infectious diseases. $4^{\text {th }}$ ed. New York: McGraw-Hill; 2004. p. 661-3.

3. Ruchel, R. Cleavage of immunoglobulines by pathogenic yeast of genus Candida. Microbial Sci. 1986; 3: 316-9.

4. Greenwood D, Richard C, Slack R, et al. Medical Microbiology. $5^{\text {th }}$ ed. London: Churchill Livingstone; 1997. p. 18-20.

5. Ellis DH. Clinical mycology - The human opportunistic mycoses. New York: Pfzor; 1994. p. 454-500.
6. McGinnis, MR. Laboratory handbook of medical mycology. New York: Academic Press; 1980. p. 11525.

7. Donghwa K, Woon S, Kyoung $\mathrm{H}$, et al. Rapid differentiation of Candida albicans from other Candida species using its unique germ tube formation. Yeast J. 2002; 19: 957-62.

8. Reischl U, Pulz $M$, Ehret $W$, et al. PCR-based detection of Mycobacteria in sputum samples using a simple and reliable DNA extraction protocol. Biotechniques. 1994; (5): 844-5.

9. Harmal NS, Khodavandi A, Mohammed A. Alshawsh $M A$, et al. Simplex and triplex polymerase chain reaction (PCR) for identification of three medically important Candida species. Afr J Biotechnol. 2012; 11(65): 12895-902.

10. Ansari Sh, Shirzadi E, Elahi M. The prevalence of fungal infections in children with hematologic malignancy in Ali-Asghar Children Hospital between 2005 and 2010. Iran J Ped Hematol Oncol. 2015; 5(1): 1-10.

11. Ramirez-Garcia A, Rementeria A, Aguirre-Urizar JM, et al. Candida albicans and cancer: Can this yeast induce cancer development or progression? Crit Rev Microbiol. 2014; 39: 1-13.

12. Faris NS. Respiratory tract bacterial infection etiological agents and susceptibility testing. Eur Scientific J. 2014; 10: 1857-81.

13. Kali A, Charles MVP, Noyal MJ, et al. Prevalence of Candida co-infection in patients with pulmonary tuberculosis. Australian Medical J. 2013; 6(8): 387-91.

14. Low $C-Y$, Rotstein C. Emerging fungal infections in immunocompromised patients. F1000 Med Rep. 2011; 3(14): 445-65.

15. Chen P, Shi M, Feng G-D, et al. A highly efficient ZiehlNeelsen stain: identifying de novo intracellular Mycobacterium tuberculosis and improving detection of extracellular M. tuberculosis in cerebrospinal fluid. J Clin Microbiol. 2012; (4): 1166-70.

16. Rajeev Shah, Parul C. Prevalence of Candida from sputum in HIV infected patients of Gujarat, India. Int J Curr Microbiol App Sci. 2014; 3(8): 345-57.

17. Cheang $P$, Chong $P$, Shamsudin $M$, et al. Cloning of phospholipase $\mathrm{B}$ gene homology in Candida species via degenerate PCR. Biomed Res. 2006; 17: 117-23.

18. Al-Rashedi N. Evaluation of Candida albicans diagnosis by using conventional PCR. J Al-Qadisiyah Pure Sci. 2012; 17: 1-10.

19. Pranab K. Jyotsna C. Duncan M. et al. Differential expression of Candida albicans phospholipase B (PLB1) under various environmental and physiological conditions. Microbiology. 2003; 149, 261-26.

\footnotetext{
Correspondence to Marwa A. Hadab E-mail: marwahadab@yahoo.com Received 25 ${ }^{\text {th }}$ Jul. 2016: Accepted $16^{\text {th }}$ Nov. 2016
} 